\title{
Multi-omics analysis of aniline-degrading bacterium, Delftia sp. K82
}

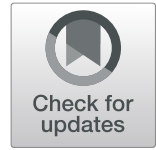

Sang-Yeop Lee ${ }^{1 \dagger}$, Sung Ho Yun ${ }^{2 \dagger}$, Hayoung Lee ${ }^{1,3}$, Giwan Seo ${ }^{1}$ and Seung $\|$ Kim $^{1,3^{*}}$

\begin{abstract}
Delftia sp. K82 is a soil bacterium capable of utilizing monocyclic aromatic hydrocarbons, including aniline, as its sole carbon and nitrogen source. In this study, the genome analysis of Delftia sp. K82 was completed and the genome data (6117 protein-coding genes and 62 RNA genes) were utilized for proteomic and transcriptomic analysis of Delftia sp. K82 cultured in aniline culture medium. Using these multi-omics approaches (genomics, transcriptomics, and proteomics), complete gene clusters for aniline biodegradation pathways were identified and transcriptomic or proteomic sets specifically induced in aniline culture conditions were elucidated. These data provide multi-layered information on the metabolic characteristics of Delftia sp. K82. The findings suggest that multi-omics approaches are useful analytical tools for the elucidation of the metabolic diversity of soil bacteria and for the identification of novel metabolic enzymes.
\end{abstract}

Keywords: Delftia sp. K82, Genomics, Transcriptomics, Proteomics, Biodegradation, Aniline

\section{Introduction}

Delftia sp. K82 was isolated from soil in the Gyeonggi province of Korea in 1992. Delftia sp. K82 has been previously known as Pseudomonas sp. K82 (Yun et al. 2004). This strain degrades monocyclic aromatic hydrocarbons (MAHs), such as aniline analogs (aniline, 3methyl aniline, and 4-methyl-aniline) and benzoate analogs (benzoate and $p$-hydroxybenzoate), as the sole carbon or nitrogen source (Yun et al. 2004).

Aniline and its analogs are toxic organic compounds and important environmental pollutants (Feng et al. 2020; Liu et al. 2002). In general, major dioxygenases for aniline (aniline analogs) biodegradation are categorized as intradiol cleavage enzymes (catechol 1,2-dioxygenase) or extradiol cleavage enzymes (catechol 2,3-dioxygenase) (Lee et al. 2016). The two types of catechol dioxygenases

\footnotetext{
* Correspondence: ksi@kbsi.re.kr

†Sang-Yeop Lee and Sung Ho Yun contributed equally to this work.

${ }^{1}$ Research Center for Bioconvergence Analysis, Korea Basic Science Institute,

Ochang 28119, South Korea

${ }^{3}$ Department of Bio-Analytical Science, University of Science and Technology

(UST), Daejeon 34113, Korea

Full list of author information is available at the end of the article
}

lead to different biodegradation pathways (Fuchs et al. 2011).

Delftia sp. K82 degrades MAHs using both types of catechol dioxygenases, and its biodegradation activity has been confirmed using two dimensional-gel-based proteomic analysis (Yun et al. 2004). In this strain, aniline-induced catechol 2,3-dioxygenase and other enzymes of the extradiol cleavage pathway as the major biodegradation pathway. Additionally, catechol 1,2-dioxygenase activities have been detected (Yun et al. 2004). However, previous gel-based proteomic analyses have not revealed enough information on the biodegradation pathway to fully understand the metabolic activities of Delftia sp. K82 (Yun et al. 2004).

Therefore, in this study, whole genome sequencing was performed to comprehensively understand the genomic characteristics for aniline biodegradation. Next, RNA sequencing (RNA-Seq) and liquid chromatography-tandem mass spectrometry (LC-MS/MS)-based proteomic analysis were performed to obtain multilayered information on aniline biodegradation activities. This omics approach determined the global response of Delftia sp. K82 to aniline culture conditions and provided complete information on the aniline 
Table 1 Genomic feature of Delftia sp. K82

\begin{tabular}{lll}
\hline Attribute & Value & \% of Total \\
\hline Genome size (bp) & $7,112,516$ & 100 \\
DNA coding (bp) & $6,251,083$ & 87.9 \\
DNA G+C (bp) & $4,667,030$ & 65.9 \\
DNA scaffolds & 21 & $\mathrm{n} / \mathrm{a}$ \\
Total genes & 6327 & 100 \\
Protein coding genes & 6117 & 96.7 \\
RNA genes & 62 & 1.0 \\
Pseudo genes & 148 & 2.3 \\
Genes in internal clusters & 723 & 11.4 \\
Genes with function prediction & 4386 & 69.3 \\
Genes assigned to COGs & 4104 & 64.9 \\
Genes with Pfam domains & 4390 & 69.4 \\
Genes with signal peptides & 1272 & 20.1 \\
Genes with transmembrane helices & 1391 & 21.9 \\
CRISPR repeats & 0 & 0 \\
\hline
\end{tabular}

biodegradation pathway. From this study, it was confirmed that Delftia sp. K82 induced two aniline degradation pathways, even though the extradiol pathway plays a major role. Other metabolic characteristics induced by aniline were also elucidated using these approaches.

In this study, it was demonstrated that multi-omics analysis is a valuable tool for the elucidation of unknown or hidden metabolic diversity of soil bacteria.

\section{Experimental}

\section{Bacterial cultivation and genomic DNA preparation}

Delftia sp. K82 was isolated as previously described (Yun et al. 2004). For extraction of genomic DNA, RNA, and proteome, Delftia sp. K82 was grown in Luria-Bertani (LB) broth at $30{ }^{\circ} \mathrm{C}$ until later part of an exponential

Table 2 Number of differentially expressed genes and proteins according omics-analysis of Delftia sp. K82

\begin{tabular}{ll}
\hline DEP(ANI/LB) & No. of proteins \\
\hline Aniline only & 472 \\
$\mid 2 f^{*} \geq 1$ & 299 \\
$1 \geq I 2 f C \leq-1$ & 1549 \\
I2fC $\leq-1$ & 438 \\
LB only & 409 \\
DEG(ANI/LB) & No. of genes \\
I2fC $\geq 1$ and $p$ value $<0.05$ & 1262 \\
I2fC $\leq-1$ and $p$ value $<0.05$ & 2657 \\
DEP and DEG & No. of proteins and genes \\
Upregulate & 195 \\
Downregulate & 442 \\
\hline
\end{tabular}

*log2 fold change phase that an optical density at $600 \mathrm{~nm}\left(\mathrm{OD}_{600}\right)$ of $0.7 \sim 0.8$. Extraction and purification of genomic DNA was performed using a G-spin DNA extraction kit (iNtRON Biotechnology Inc., Sungnam, Korea), according to the manufacturer's instructions. Delftia sp. K82 cultured in aniline media was prepared as previously described (Yun et al. 2004). Briefly, aniline media was composed of potassium phosphate buffer ( $\mathrm{pH}$ 6.25) containing $3.4 \mathrm{mM} \mathrm{MgSO}_{4}, 0.3 \mathrm{mM} \mathrm{FeSO}_{4}, 0.2 \mathrm{mM} \mathrm{CaCO}_{3}$, $10 \mathrm{mM} \mathrm{NH}_{4} \mathrm{Cl}$, and $5 \mathrm{mM}$ aniline.

\section{Genome sequencing and assembly}

The Delftia sp. K82 genome was sequenced on an Illumina MiSeq platform (Illumina, San Diego, CA, USA) using a paired-end and a mate-pair strategy. Paired-end and mate-pair libraries were created with insert sizes of 250-350 bp and $5 \mathrm{~kb}$, respectively. Low-quality reads were trimmed using PRINSEQ lite (Schmieder and Edwards 2011). De-novo assembly and construction of the draft genome of Delftia sp. K82 was performed with 41, 757,452 high-quality reads using CLC Genomics Workbench v7.0 (QIAGEN Bioinformatics, Germantown, MD, USA).

\section{Genome annotation}

Genome annotation was performed using Prokka 2.6.0, with the Delftia genus protein database downloaded from Uniprot and the Rapid Annotation using Subsystem Technology (RAST) annotation server (Seemann 2014; Aziz et al. 2008). Pairwise comparisons were performed for validation of predicted open reading frames. The rRNA and tRNA genes were detected using RNAmmer and tRNAscan-SE, respectively (Lagesen et al. 2007; Chan and Lowe 2019). Genes in internal clusters were identified using BLASTclust with thresholds of $>70 \%$ coverage length and $>30 \%$ sequence identity. Transmembrane helices, signal peptides, and clustered regularly interspaced short palindromic repeats (CRISPR) were analyzed using TMHMM, signalP, and CRISPRfinder, respectively (Chen et al. 2003; Almagro Armenteros et al. 2019; Grissa et al. 2007).

\section{LC-MS/MS analysis (proteomic analysis)}

To extract total protein samples, harvested bacteria were boiled in 10\% sodium dodecyl sulfate (SDS) for $10 \mathrm{~min}$, and the supernatant was obtained by centrifugation (15, $000 \times g$ for $10 \mathrm{~min})$. Extracted proteins were then fractionated using 12\% SDS-polyacrylamide gel electrophoresis. Tryptic digestion was performed, as previously described (Yun et al. 2011b). Tryptic peptides were dissolved with $0.5 \%$ trifluoroacetic acid prior to further analysis. Concentrated tryptic peptides were directed onto a $10 \mathrm{~cm} \times 75 \mu \mathrm{m}$ ID C18 reverse-phase column at a flow rate of $300 \mathrm{~nL} / \mathrm{min}$ and were eluted with a gradient of 


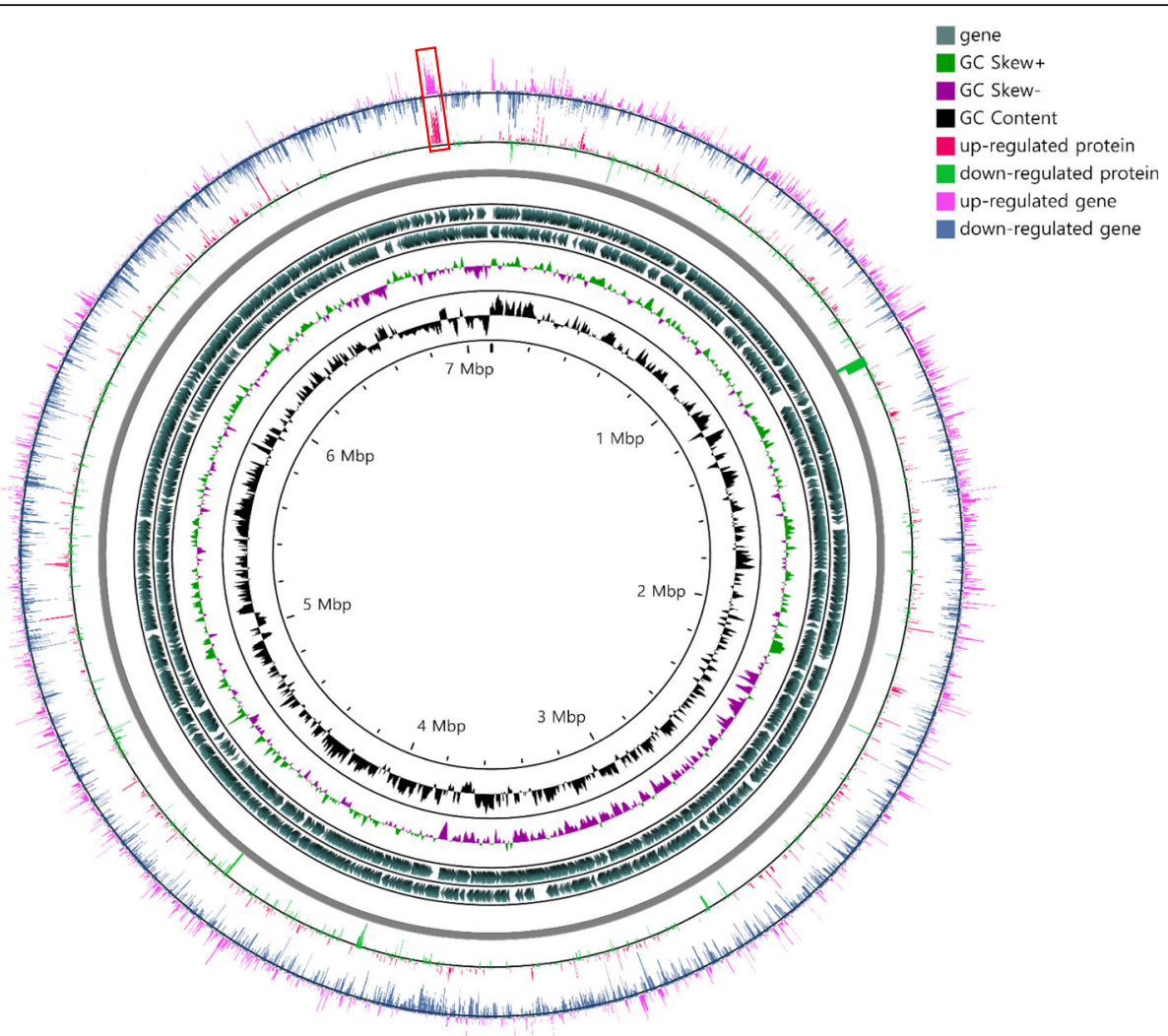

Fig. 1 Multi-layered omics information of Delftia sp. K82. The inner black circle indicates GC content, and the green and purple circles represent the GC skew of Delftia sp. K82 genome. The first and second outer rings show the log 2 fold change values for transcriptomics and proteomics when compared to aniline and LB conditions, respectively. The location of the aniline degradation cluster is indicated by a red box

$8-55 \%$ acetonitrile over $80 \mathrm{~min}$. All MS and MS/MS spectra were acquired on an LTQ-Velos electrospray ionization ion trap mass spectrometer (Thermo Scientific, Waltham, MA, USA) in data-dependent mode. MASCOT (v.2.4) was used for protein identification. To quantitatively compare the protein abundance in each sample, differentially expressed proteins (DEPs) were calculated using the mol\% value.

\section{RNA-Seq and bioinformatics analysis}

RNA isolation was performed using the RNeasy Plus Mini Kit (QIAGEN, Valencia, CA, USA), and RNA quality was confirmed using the Agilent RNA 6000 Nano kit (Agilent Technologies, Santa Clara, CA, USA). Magnetic beads conjugated to oligo $(\mathrm{dT})$ were used to enrich poly (A) mRNA before cDNA library construction. The final library sizes and qualities were evaluated electrophoretically using an Agilent High Sensitivity DNA kit (Agilent Technologies). Subsequently, the library was sequenced on an Illumina NovaSeq6000 sequencer (Illumina, San Diego, CA, USA). Low-quality reads and adapter sequences were filtered out using Trimmomatic (v.0.32) (Bolger et al. 2014). Filtered reads were aligned to the Delftia sp. K82 genome (Accession: NZ
MYFL00000000.1) using STAR (v.2.3.0) (Dobin et al. 2013). The number of mapped sequences on genes was calculated using HTSeq-count (v.0.5.4) (Anders et al. 2015), and differentially expressed genes (DEGs) were identified using the $\mathrm{R}$ package DESeq2 (Love et al. 2014). Functional annotation of DEGs were analyzed using GSEA-Pro v.3 (http://gseapro.molgenrug.nl/), and the CGView server was used to construct the circular map (Grant and Stothard 2008).

\section{Results and discussion}

Summary of genomic, transcriptomic, and proteomic analyses

The genome of Delftia sp. K82 consisted of 21 scaffolds, and the total length of the scaffolds was 7,112,516 bp, with an average GC content of $65.9 \%$. Delftia sp. K82 had 6327 genes. Among them, 6117 genes (96.7\%) were protein-coding genes. A total of 4104 genes of the protein-coding sequence (64.9\%) were assigned to Clusters of Orthologous Group categories and 4386 (69.3\%) were annotated as putative proteins. Genome data were deposited in the National Center for Biotechnology Information (NZ_MYFL00000000.1). Table 1 provides a more detailed summary of the genome properties. 


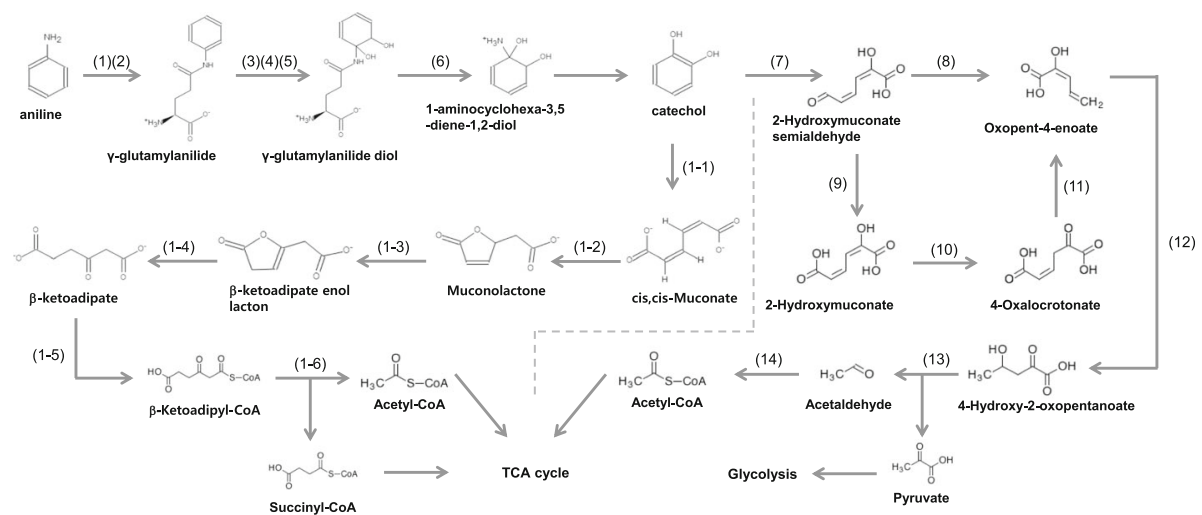

intradiol cleavage pathway

extradiol cleavage pathway

Fig. 2 Two metabolic pathway of aniline degradation in Delftia sp. K82. Two metabolic pathways (an intradiol cleavage pathway and an extradiol cleavage pathway) of aniline degradation in Delftia sp. K82 have been identified. Enzymes of each pathway are numbered and are listed in Table 3. The aniline oxygenase complex of Delftia sp. K82 is composed of six enzymes (Nos. 1 6) and four reaction steps for the conversion of aniline into catechol. The locus_tag and enzyme name of each number are as follows: No.1, KDK82_RS31140, glutamine synthetase; No.2,KDK82_RS31135,glutamine amidotransferase ; No.3,KDK82_RS31130,Large subunit of dioxygenase; No.4,KDK82_RS31125,small subunit of dioxygenase ; No.5,KDK82_RS31120,aniline dioxygenase reductase; No.6,KDK82_RS31110,small ferredoxin-like protein ; No.7,KDK82_RS31105, catechol 2,3-dioxygenase; No.8,KDK82_RS31090, 2hydroxymuconic semialdehyde hydrolase; No.9, KDK82_RS31095, 2-hydroxymuconic semialdehyde dehydrogenase; No.10, KDK82_RS31050, 4oxalocrotonate tautomerase family protein; No.11, KDK82_RS31080, 2-oxo-3-hexenedioate decarboxylase; No.12, KDK82_RS31085, 2-oxopent-4-enoate hydratase; No.13, KDK82_RS31060, 4-hydroxy-2-oxovalerate aldolase; No.14, KDK82_RS31065, acetaldehyde dehydrogenase; No.1-1, KDK82_RS22950, catechol 1,2-dioxygenase; No.1-2, KDK82_RS22965, mandelate racemase; No.1-3,KDK82_RS22960, muconolactone delta-isomerase; No.1-4, KDK82_RS23310, 3-oxoadipate enol-lactonase; No.1-5, KDK82_RS01060, 3-oxoadipate CoA-transferase; No.1-6, KDK82_RS17030, acetyl-CoA C-acyltransferase

Table 3 Results of multi-omics analysis of aniline degradation pathway in Delftia sp. K82

\begin{tabular}{|c|c|c|c|c|}
\hline \multirow[t]{2}{*}{ Index } & \multirow[t]{2}{*}{ Locus_tag } & \multirow[t]{2}{*}{ Product } & \multicolumn{2}{|c|}{ log2 Fold change (ANI/LB) } \\
\hline & & & Transcriptome & Proteome \\
\hline 1 & KDK82_RS31140 & Glutamine synthetase & 3.631 & 7.356 \\
\hline 2 & KDK82_RS31135 & Glutamine amidotransferase & 3.129 & 4.650 \\
\hline 3 & KDK82_RS31130 & Large subunit of dioxygenase & 3.492 & 8.566 \\
\hline 4 & KDK82_RS31125 & Small subunit of dioxygenase & 2.907 & 3.552 \\
\hline 5 & KDK82_RS31120 & Aniline dioxygenase reductase & 4.322 & 6.663 \\
\hline 6 & KDK82_RS31110 & Small ferredoxin-like protein & 1.322 & ANI only \\
\hline 7 & KDK82_RS31105 & Catechol 2,3-dioxygenase & 6.809 & 5.952 \\
\hline 8 & KDK82_RS31090 & 2-Hydroxymuconic semialdehyde hydrolase & 3.907 & 7.057 \\
\hline 9 & KDK82_RS31095 & 2-Hydroxymuconic semialdehyde dehydrogenase & 5.229 & 5.356 \\
\hline 10 & KDK82_RS31050 & 4-Oxalocrotonate tautomerase family protein & 6.229 & 6.584 \\
\hline 11 & KDK82_RS31080 & 2-Oxo-3-hexenedioate decarboxylase & 5.322 & 6.466 \\
\hline 12 & KDK82_RS31085 & 2-Oxopent-4-enoate hydratase & 4.269 & 4.831 \\
\hline 13 & KDK82_RS31060 & 4-Hydroxy-2-oxovalerate aldolase & 8.492 & 7.649 \\
\hline 14 & KDK82_RS31065 & Acetaldehyde dehydrogenase & 6.662 & 6.733 \\
\hline $1-1$ & KDK82_RS22950 & Catechol 1,2-dioxygenase & 1.100 & 4.427 \\
\hline $1-2$ & KDK82_RS22965 & Mandelate racemase & 0.907 & ANI only \\
\hline $1-3$ & KDK82_RS22960 & Muconolactone delta-isomerase & -1.121 & 0.942 \\
\hline $1-4$ & KDK82_RS23310 & 3-Oxoadipate enol-lactonase & -3.121 & -0.199 \\
\hline $1-5$ & KDK82_RS01060 & 3-Oxoadipate CoA-transferase & -3.059 & ANI only \\
\hline $1-6$ & KDK82_RS17030 & Acetyl-CoA C-acyltransferase & -0.858 & ANI only \\
\hline
\end{tabular}




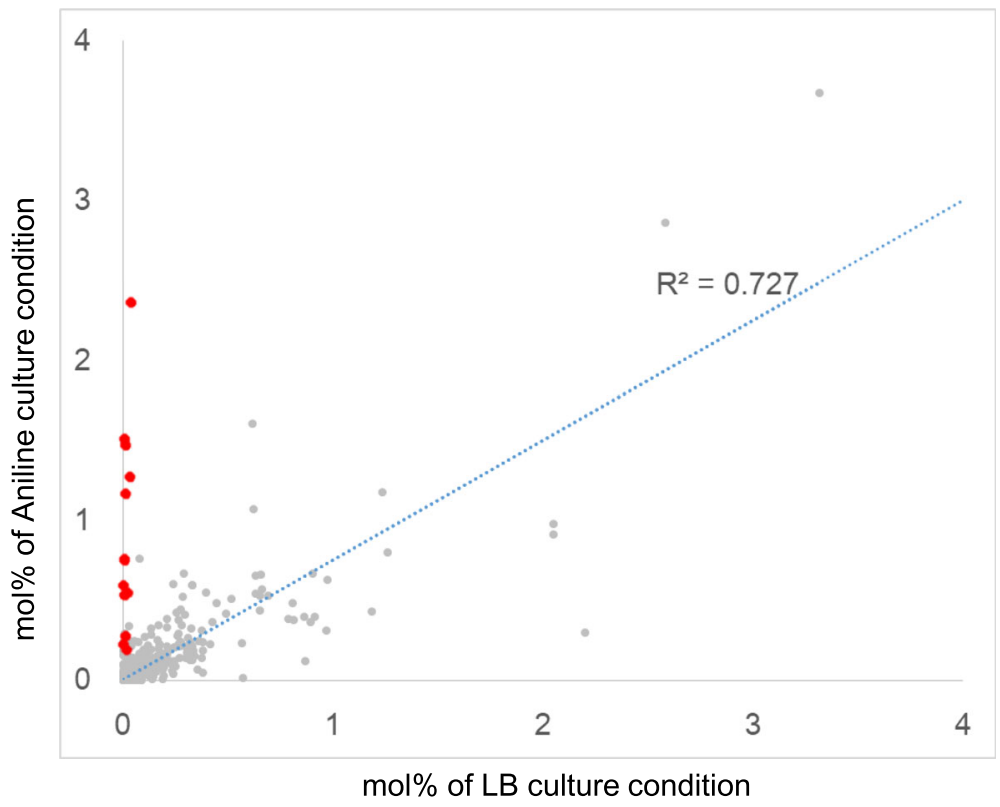

Fig. 3 Correlation of two proteome sets of Delftia sp. K82. The two proteome sets are highly correlated $\left(R^{2}: 0.727\right)$ when aniline degradation cluster proteins are excluded. Red dots indicate aniline-degrading cluster proteins. However, when aniline degradation-related proteins are included, $r$ squared value of two proteome sets was decreased to 0.519

For RNA-Seq and proteomic analysis using LC/MSMS analysis, two cultures (LB media and aniline media) of Delftia sp. K82 were prepared, according to a previously described procedure (Yun et al. 2004). The results of transcriptomic and proteomic analyses are summarized in Table 2. A total of 3919 genes $(64.07 \%$ of the total protein-coding genes) and 1618 genes $(26.4 \%$ of the total protein-coding genes) were identified as differentially expressed genes (abs (log2 fold change) $\geq 1$ ) in the transcriptomic and proteomic analyses, respectively. Figure 1 shows the genome-wide multilayered omics results (genomics, transcriptomics, and proteomics) of Delftia sp. K82.

\section{Characterization of the aniline degradation pathway of Delftia sp. $\mathrm{K} 82$ at the level of multi-omics analysis}

According to the genomic analysis of Delftia sp. K82, Delftia sp. K82 had two different aniline degradation pathways (intradiol cleavage and extradiol cleavage). In a previous study, we suggested that Delftia sp. K82 induced two cleavage activities and reported a partial amino acid sequence of two pathway enzymes (Yun et al. 2004). However, we could not confirm the complete gene information of aniline degradation pathways because of the lack of a whole-genome sequence. Therefore, the whole genome sequence of Delftia sp. K82 was constructed in this study and the aniline degradation cluster was identified in the genome sequence. As a result, two complete aniline degradation pathways were obtained (Fig. 2). Their transcriptomic and proteomic induction levels are summarized in Table 3. The aniline oxygenase complex of Delftia sp. K82 (Nos. 1 6), converting aniline into catechol, and the extradiol cleavage pathway (Nos. 7 14) were highly induced more than 2-fold change in aniline condition at the transcriptional and translational levels (Table 3). However, induction of the intradiol cleavage pathway (Nos. 1-2 1-6), except for catechol 1,2-dioxygenase (No. 1-1) was less than 2-fold change or down-expressed in aniline condition. From these results, it was assumed that the extradiol cleavage of aniline was a major metabolic pathway. However, the role of the intradiol cleavage pathway of Delftia sp. K82 was not clear, but it seems to be complementary in the utilization of aniline.

\section{Genome-wide characterization of aniline-induced high- copy metabolic enzymes of Delftia sp. K82}

Semi-quantitative data of the whole proteome were obtained through spectral counts or mol\% calculation using LC-MS/MS analysis (Ishihama et al. 2005). These data will be valuable for understanding the cell-based physiological status of bacteria at the proteomic level. For this purpose, 276 highly abundant proteins were selected from Delftia sp. K82 (90th percentile of mol\%) for this analysis (Supplementary data 1). These abundant proteins were estimated to be approximately $71.5 \%$ of the total amount of whole proteins. For Delftia sp. K82 cultured in aniline, amount of proteins expression of 14 aniline degradation enzymes composed up to $11.9 \%$ of all proteins. Compared to Delftia sp. K82 cultured in LB 
(less than $0.17 \%$ aniline degradation enzymes), the induction of aniline degradation enzymes was very tightly regulated in the presence of aniline in the culture media. However, significant variation in other basic metabolic enzymes, such as the tricarboxylic acid cycle, protein synthesis, and amino acid metabolism, were not identified (Fig. 3). This result indicated that Delftia sp. K82 makes essential metabolic enzymes regardless of the nutrition source provided. However, there were several upregulated abundant proteins in the aniline culture (Table $4)$.

\section{Genome-wide characterization of aniline-induced cell wall and membrane proteins of Delftia sp. K82}

Cell wall or membrane proteins play important roles in transport, signal transduction, and protection against extracellular stress (Park et al. 2012; Yun et al. 2011a). In the case of Delftia sp. K82, identification of anilineinduced cell wall and membrane proteins was important to elucidate the biodegradation activities of Delftia sp. K82. PSORTb was used to predict the subcellular location of induced proteins (Yu et al. 2010). Among the 95 proteins belonging to the cell wall and membrane fraction of the 276 highly abundant proteins of Delftia sp. K82, 12 proteins were identified that were significantly induced in aniline media (Table 4). Specifically, it is possible that porins (KDK82-RS00485) and transporters (KDK82-RS27635, RS31055, RS22955, RS00520, and RS01045) could be related to the transport of aniline or related metabolites. However, to fully understand the upregulated cell wall or membrane proteins, further functional studies, such as targeted mutagenesis, are required.

\section{Conclusions}

Multi-omics analysis of Delftia sp. K82 was performed, and it revealed the genome-wide response of Delftia sp. K82 to aniline. Both enzymes of the aniline degradation pathway and also aniline-induced novel proteins were identified. These results suggest that multi-omics approaches are useful tools for screening novel genes for a biodegradation response (Horinouchi et al. 2018; Yang et al. 2016). In particular, this approach can be applied to the elucidation of bacteria with diverse activities for organic compounds and the identification of novel enzymes for biodegradation (Swanson 1999). These enzymes can be used for the production of high-value added metabolites, which are used as precursors for biotechnological or pharmaceutical products (Hegazy et al. 2015; Zaks 2001).

Table 4 Upregulated abundant proteins in aniline culture condition except aniline degradation pathway

\begin{tabular}{|c|c|c|c|c|c|}
\hline \multirow[t]{2}{*}{ Locus_tag } & \multirow[t]{2}{*}{ Product } & \multirow[t]{2}{*}{ Localization } & \multirow{2}{*}{$\begin{array}{l}\text { log2 Fold change } \\
\text { (ANI/LB) }\end{array}$} & \multicolumn{2}{|l|}{$\mathrm{mol} \%$} \\
\hline & & & & LB & ANI \\
\hline KDK82_RS01055 & 3-Oxoadipyl-CoA thiolase & Cytoplasmic & 3.569 & 0.0287 & 0.3406 \\
\hline KDK82_RS28665 & Succinate dehydrogenase iron-sulfur subunit & Cytoplasmic & 1.735 & 0.0717 & 0.2386 \\
\hline KDK82_RS31120 & Hypothetical protein & Cytoplasmic & 6.663 & 0.0023 & 0.233 \\
\hline KDK82_RS06005 & D-threitol dehydrogenase & Cytoplasmic & 2.414 & 0.0398 & 0.2121 \\
\hline KDK82_RS00570 & DUF1338 domain-containing protein & Cytoplasmic & 5.790 & 0.0034 & 0.1881 \\
\hline KDK82_RS31100 & Heme-binding protein & Cytoplasmic & 6.047 & 0.0027 & 0.1785 \\
\hline KDK82_RS14040 & Copper chaperone PCu(A)C & Cytoplasmic & 2.310 & 0.0196 & 0.0972 \\
\hline KDK82_RS05985 & Dihydroxyacetone kinase subunit $\mathrm{L}$ & Cytoplasmic & 1.396 & 0.0348 & 0.0916 \\
\hline KDK82_RS00635 & Aldehyde dehydrogenase & Cytoplasmic & 1.916 & 0.0226 & 0.0853 \\
\hline KDK82_RS09610 & Hcp1 family type VI secretion system effector & Extracellular & 2.397 & 0.0431 & 0.227 \\
\hline KDK82_RS00485 & Porin & Outer membrane & 3.116 & 0.028 & 0.2428 \\
\hline KDK82_RS08655 & TonB-dependent receptor & Outer membrane & 5.814 & 0.0033 & 0.1857 \\
\hline KDK82_RS27635 & Branched-chain amino acid $A B C$ transporter substrate-binding protein & Periplasmic & 1.287 & 0.1332 & 0.325 \\
\hline KDK82_RS31055 & Tripartite tricarboxylate transporter substrate binding protein & Periplasmic & 5.049 & 0.0088 & 0.2914 \\
\hline KDK82_RS22955 & Tripartite tricarboxylate transporter substrate binding protein & Periplasmic & 7.762 & 0.0011 & 0.2387 \\
\hline KDK82_RS31125 & DcaA2 & Periplasmic & 3.552 & 0.017 & 0.1994 \\
\hline KDK82_RS03245 & NADP-specific glutamate dehydrogenase & Periplasmic & 2.153 & 0.0234 & 0.1041 \\
\hline KDK82_RS00520 & Amino acid $A B C$ transporter substrate-binding protein & Periplasmic & 6.395 & 0.0012 & 0.101 \\
\hline KDK82_RS01045 & Tripartite tricarboxylate transporter substrate binding protein & Periplasmic & 3.015 & 0.0122 & 0.0986 \\
\hline KDK82_RS04080 & DUF1993 domain-containing protein & Periplasmic & 1.333 & 0.0375 & 0.0945 \\
\hline KDK82_RS20410 & C4-dicarboxylate $A B C$ transporter & Periplasmic & 2.248 & 0.0173 & 0.0822 \\
\hline
\end{tabular}




\section{Supplementary Information}

The online version contains supplementary material available at https://doi. org/10.1186/s40543-021-00258-6.

Additional file 1: Supplementary Table 1. High abundant proteins (90th percentile of mol\%) in aniline culture condition of Delftia sp. K82.

\section{Abbreviations}

MAH: Mono aromatic hydrocarbon; LC-MS/MS: Liquid chromatography-mass spectrometry; LB: Luria-Bertani; PSORTb: Protein subcellular localization predictions; RSSR: Rapid Annotation using Subsystem Technology; COG: Clusters of orthologous group; DEG: Differentially expressed gene; DEP: Differentially expressed protein; CRISPER: Clustered regularly interspaced short palindromic repeats

\section{Acknowledgements}

Not applicable.

\section{Availability of supporting data}

Research data have been provided in the manuscript.

\section{Authors' contributions}

SIK and SYL performed data analysis. HYL, GWS, and SHY carried out all experimental work. SIK designed and wrote the manuscript. All authors read and approved the final manuscript.

\section{Funding}

This work was supported by the Korea Basic Science Institute research program (No. C030130 and C030222), and the National Research Foundation of Korea (NRF) grant funded by the Korea government (MSIT) (No. 2020R1C1C1009941)

\section{Competing interests}

The authors declare that they have no competing interests.

\section{Author details}

${ }^{1}$ Research Center for Bioconvergence Analysis, Korea Basic Science Institute, Ochang 28119, South Korea. ${ }^{2}$ Center for Research Equipment, Korea Basic Science Institute, Ochang 28119, South Korea. ${ }^{3}$ Department of Bio-Analytical Science, University of Science and Technology (UST), Daejeon 34113, Korea.

\section{Received: 6 October 2020 Accepted: 13 January 2021}

Published online: 05 February 2021

\section{References}

Almagro Armenteros JJ, Tsirigos KD, Sonderby CK, et al. SignalP 5.0 improves signal peptide predictions using deep neural networks. Nat Briotechnol. 2019;37(4):420-3. https://doi.org/10.1038/s41587-019-0036-z.

Anders S, Pyl PT, Huber W. HTSeq-a Python framework to work with highthroughput sequencing data. Bioinformatics. 2015;31(2):166-9. https://doi. org/10.1093/bioinformatics/btu638.

Aziz RK, Bartels D, Best AA, et al. The RAST Server: rapid annotations using subsystems technology. BMC Genomics. 2008;9:75. https://doi.org/10.1186/ 1471-2164-9-75

Bolger AM, Lohse M, Usadel B. Trimmomatic: a flexible trimmer for Illumina sequence data. Bioinformatics. 2014;30(15):2114-20. https://doi.org/10.1093/ bioinformatics/btu170.

Chan PP, Lowe TM. tRNAscan-SE: searching for tRNA genes in genomic sequences. Methods Mol Biol. 2019;1962:1-14. https://doi.org/10.1007/978-14939-9173-0_1.

Chen Y, Yu P, Luo J, Jiang Y. Secreted protein prediction system combining CJSPHMM, TMHMM, and PSORT. Mammalian Genome. 2003;14(12):859-65. https://doi.org/10.1007/s00335-003-2296-6.

Dobin A, Davis CA, Schlesinger F, et al. STAR: ultrafast universal RNA-seq aligner. Bioinformatics. 2013;29(1):15-21. https://doi.org/10.1093/bioinformatics/ bts635.

Feng $Y$, Chen $L L$, Zhang $T$, Wu $Y H$, Song $L$, Wang $Y$. Research progress on health effects of aniline. Zhonghua Yu Fang Yi Xue Za Zhi. 2020;54(2):213-8. https:// doi.org/10.3760/cma.j.issn.0253-9624.2020.02.019.
Fuchs G, Boll M, Heider J. Microbial degradation of aromatic compounds-from one strategy to four. Nat Rev Microbiol. 2011;9(11):803-16. https://doi.org/10. 1038/nrmicro2652.

Grant JR, Stothard P. The CGView Server: a comparative genomics tool for circular genomes. Nucleic Acids Res. 2008;36(Web Server issue):W181-4. https://doi.org/10.1093/nar/gkn179.

Grissa I, Vergnaud G, Pourcel C. CRISPRFinder: a web tool to identify clustered regularly interspaced short palindromic repeats. Nucleic Acids Res. 2017; 35(Web Server issue):W52-57. https://doi.org/10.1093/nar/gkm360.

Hegazy ME, Mohamed TA, ElShamy Al, et al. Microbial biotransformation as a tool for drug development based on natural products from mevalonic acid pathway: a review. J Adv Res. 2015;6(1):17-33. https://doi.org/10.1016/j.jare. 2014.11.009.

Horinouchi T, Maeda T, Furusawa C. Understanding and engineering alcoholtolerant bacteria using OMICS technology. World J Microbiol Biotechnol. 2018:34(11):157. https://doi.org/10.1007/s11274-018-2542-4.

Ishihama Y, Oda Y, Tabata T, et al. Exponentially modified protein abundance index (emPAl) for estimation of absolute protein amount in proteomics by the number of sequenced peptides per protein. Mol Cell Proteomics. 2005; 4(9):1265-72. https://doi.org/10.1074/mcp.M500061-MCP200.

Lagesen K, Hallin P, Rodland EA, Staerfeldt HH, Rognes T, Ussery DW. RNAmmer: consistent and rapid annotation of ribosomal RNA genes. Nucleic Acids Res. 2007;35(9):3100-8. https://doi.org/10.1093/nar/gkm160.

Lee SY, Kim GH, Yun SH, et al. Proteogenomic characterization of monocyclic aromatic hydrocarbon degradation pathways in the aniline-degrading bacterium burkholderia sp. K24. PloS One. 2016;11(4):e0154233. https://doi. org/10.1371/journal.pone.0154233.

Liu Z, Yang H, Huang Z, Zhou P, Liu SJ. Degradation of aniline by newly isolated, extremely aniline-tolerant Delftia sp. AN3. Appl Microbiol Biotechnol. 2002; 58(5):679-82. https://doi.org/10.1007/s00253-002-0933-8.

Love MI, Huber W, Anders S. Moderated estimation of fold change and dispersion for RNA-seq data with DESeq2. Genome Biol. 2014;15(12):550. https://doi.org/10.1186/s13059-014-0550-8.

Park JS, Lee WC, Yeo KJ, et al. Mechanism of anchoring of OmpA protein to the cell wall peptidoglycan of the gram-negative bacterial outer membrane. FASEB J. 2012;26(1):219-28. https://doi.org/10.1096/fj.11-188425.

Schmieder R, Edwards R. Quality control and preprocessing of metagenomic datasets. Bioinformatics. 2011;27(6):863-4. https://doi.org/10.1093/ bioinformatics/btr026.

Seemann T. Prokka: rapid prokaryotic genome annotation. Bioinformatics. 2014; 30(14):2068-9. https://doi.org/10.1093/bioinformatics/btu153.

Swanson PE. Dehalogenases applied to industrial-scale biocatalysis. Curr Opin Biotechnol. 1999;10(4):365-9. https://doi.org/10.1016/S0958-1669(99)80066-4.

Yang FC, Chen YL, Tang SL, et al. Integrated multi-omics analyses reveal the biochemical mechanisms and phylogenetic relevance of anaerobic androgen biodegradation in the environment. ISME J. 2016;10(8):1967-83. https://doi. org/10.1038/ismej.2015.255.

Yu NY, Wagner JR, Laird MR, et al. PSORTb 30: improved protein subcellular localization prediction with refined localization subcategories and predictive capabilities for all prokaryotes. Bioinformatics. 2010;26(13):1608-15. https:// doi.org/10.1093/bioinformatics/btq249.

Yun SH, Choi CW, Kwon SO, et al. Quantitative proteomic analysis of cell wall and plasma membrane fractions from multidrug-resistant Acinetobacter baumannii. J Proteome Res. 2011 a;10(2):459-69. https://doi.org/10.1021/ pr101012s

Yun SH, Park GW, Kim JY, et al. Proteomic characterization of the Pseudomonas putida KT2440 global response to a monocyclic aromatic compound by iTRAQ analysis and 1DE-MudPIT. J Proteomics. 2011b;74(5):620-8. https://doi. org/10.1016/j.jprot.2011.01.020.

Yun SH, Yun CY, Kim SI. Characterization of protocatechuate 4,5-dioxygenase induced from p-hydroxybenzoate-cultured Pseudomonas sp. K82. J Microbiol. 2004:42(2):152-5

Zaks A. Industrial biocatalysis. Curr Opin Chem Biol. 2001;5(2):130-6. https://doi. org/10.1016/s1367-5931(00)00181-2.

\section{Publisher's Note}

Springer Nature remains neutral with regard to jurisdictional claims in published maps and institutional affiliations. 